\title{
Indices for Assessing Utility Distribution System RMS Variation Performance
}

\author{
D. L. Brooks, R. C. Dugan \\ Members, IEEE \\ Electrotek Concepts, Inc. \\ Knoxville, Tennessee
}

\author{
Marek Waclawiak \\ Member, IEEE \\ United Illuminating Co. \\ New Haven, CT
}

\author{
Ashok Sundaram \\ Member, IEEE \\ Electric Power Research Institute \\ Palo Alto, California
}

\begin{abstract}
For many years, electricity distribution companies have used sustained interruption indices as indicators of the reliability of service provided on their systems. Today, however, many electricity consumers are adversely affected by more subtle voltage disturbances such as sags and swells. Many utilities are well aware of such service quality concerns and are implementing extensive monitoring systems to detect such disturbances and assess service quality in this regard. This paper presents a subset of work completed which provides utilities with tools to make more complete service quality assessments. Indices developed to reflect system service quality with respect to all $\mathrm{rms}$ variations are presented. Example values for the indices are calculated using data from a national distribution power quality data collection project. Finally, an example application of the indices currently being made by a distribution utility is discussed.
\end{abstract}

\section{INTRODUCTION}

The electric power industry is continuing to evolve into a more competitive marketplace as deregulation is implemented in the various states. Furthermore, the service requirements of many electric power consumers are continuing to rise. As a result of these influences, a greater emphasis than ever before is being placed on the quality of service provided to customers. A significant number of utilities have begun to apply extensive service quality monitoring systems throughout their distribution systems in order to determine the typical level of service quality provided.

Many customers differentiate service solely on price and have only low to moderate expectations with regard to the

quality of service received. Most utilities provide a baseline of service which is already acceptable to these customers.
An increasing number of customers, however, utilize enduse devices which are more sensitive to smaller perturbations in the power supply. These sensitive customers range from large industrial facilities with ASDdriven processes to residential customers with home computers. In addition to providing the baseline level of service to the low-cost customers, many utilities have realized the need, as well as the opportunity, to provide higher grades of service to those customers with extremely sensitive electrical equipment. Many premium service contracts such as the one entered into by Detroit Edison and its three largest automobile manufacturing customers have already been implemented. Many utilities are also applying service quality monitoring to mediate such premium service contracts or to provide other special services to important customers.

Whether assessing the aggregate system service quality or an individual customer service quality, utilities must quantify the raw measured data in a meaningful manner. In the past utilities have assessed service quality using sustained interruption indices such as SAIFI and CAIDI, which have become known as reliability indices. The evolution and proliferation of power electronic devices and other sensitive end-use equipment, however, have altered the reality of what is actually reliable service. Due to the sensitivity of many industrial and commercial loads such as adjustable speed drives (ASDs) and computer-controlled processes, reliability is no longer indicated by the frequency and duration of sustained interruptions occurring on the distribution system. For example, to a textile manufacturer whose main process is driven by an ASD, a six-cycle voltage sag to $80 \%$ of the nominal voltage may be just as costly in terms of lost productivity as a two-hour interruption of service. Using the traditional reliability indices to assess quality of service provided to this textile manufacturer, this costly disturbance would be neglected.

\section{CURRENT SERVICE QUALITY INDICES}

For many years, the only indices defined to quantify rms variation service quality were the sustained interruption indices (SAIFI, CAIDI, etc.). Sustained interruptions are in fact only one type of rms variation. IEEE Standard 11591995 defines a sustained interruption as a reduction in the rms voltage to less than $10 \%$ of nominal voltage for longer than 1 minute [1]. Sustained interruptions are of high 
importance because all customers on the faulted section are affected by such disturbances. Consequently, there are many indices defined to assess sustained interruptions. These indices have been used by utilities for years, although they are not yet standardized by the IEEE. They are included in the proposed IEEE Standard P1366 [2] which should go to ballot by the end of 1996 . Yet, the definition of a sustained interruption upon which the Std. P1366 indices are based is not consistent with the IEEE Std. 1159 definition which creates some confusion.

IEEE P1366 also defines indices quantifying momentary interruption performance[2]. These indices provide utilities with tools for assessing another very important type of rms variations. Momentary interruptions affect many customer types. Unfortunately, as with sustained interruptions, the definition of a momentary interruption used in Std. P1366 is not consistent with the Std. 1159 definition.

\section{CHARACTERIZATION OF RMS VARIATIONS UPON WHICH SERVICE QUALITY INDICES ARE BASED}

IEEE Standard 1159-1995, Recommended Practice on Monitoring Electric Power Quality, provides a common terminology that can be used to discuss and assess rms voltage variations. This standard defines magnitude ranges for sags, swells and interruptions. Also, 1159 suggests that the terms sag, swell, and interruption be preceded by a modifier describing the duration of the event (instantaneous, momentary, temporary, or sustained). Table 1 shows the Std. 1159 definitions of the different rms variation types.

\begin{tabular}{|c|l|l|}
\hline Category & \multicolumn{1}{|c|}{ Duration } & Voltage Magnitude \\
\hline Short Duration Variations & & \\
\hline \hline Instantaneous & & \\
\hline Sag & 0.5 to 30 cycles & 0.1 to $0.9 \mathrm{pu}$ \\
\hline Swell & 0.5 to 30 cycles & 1.1 to $1.8 \mathrm{pu}$ \\
\hline Momentary & & \\
\hline Interruption & 0.5 cycles to $3 \mathrm{~s}$ & $<0.1 \mathrm{pu}$ \\
\hline Sag & 30 cycles to $3 \mathrm{~s}$ & 0.1 to $0.9 \mathrm{pu}$ \\
\hline Swell & 30 cycles to $3 \mathrm{~s}$ & 1.1 to $1.8 \mathrm{pu}$ \\
\hline Interruption & & \\
\hline Sag & $3 \mathrm{~s}$ to 1 min & $<0.1 \mathrm{pu}$ \\
\hline Swell & $3 \mathrm{~s}$ to 1 min & 0.1 to $0.9 \mathrm{pu}$ \\
\hline Longorary Duration Variations & $3 \mathrm{~s}$ to 1 min & 1.1 to $1.8 \mathrm{pu}$ \\
\hline Sustained Interruption & & \\
\hline Undervoltage & $>1$ min & $0.0 \mathrm{pu}$ \\
\hline Overvoltage & $>1$ min & 0.8 to $0.9 \mathrm{pu}$ \\
\hline & $>1$ min & 1.1 to $1.2 \mathrm{pu}$ \\
\hline & &
\end{tabular}

Table 1. Frequency envelope plot recorded in the affected region during the contingency.

As Table 1 indicates, rms variations are classified by the magnitude and duration of the disturbances. Before rms variation indices can be calculated, characteristics such as magnitude and duration must be determined for each of the many rms variations recorded. Characterization is the process of extracting useful pieces of information from a measurement which describe the event without having to retain every detail of the event. Characterization of rms variations can be very complicated as evidenced by the literature $[3,4]$ and the IEEE PES Task Force dedicated to the subject. The following describes how rms variations are characterized for quantification by the developed indices.

Characterization of rms voltage variations is structured into three levels, each of which is identified as a type of event as follows: (1) phase or component event, (2), measurement event, and (3) aggregate event.

Component Event Level. Each phase of each rms variation measurement may contain multiple components. Most rms variations are rectangular in shape being accurately characterized by a single magnitude and duration [3,4]. Consequently, these phase measurements are easily characterized with respect to magnitude and duration. Approximately $10 \%$ of rms variations are non-rectangular [3], however, like the variation shown in Figure 1. These variations are much more difficult to characterize because no single magnitude-duration pair completely represent the phase measurement. Thus, any characterization of nonrectangular variations requires an approximation.

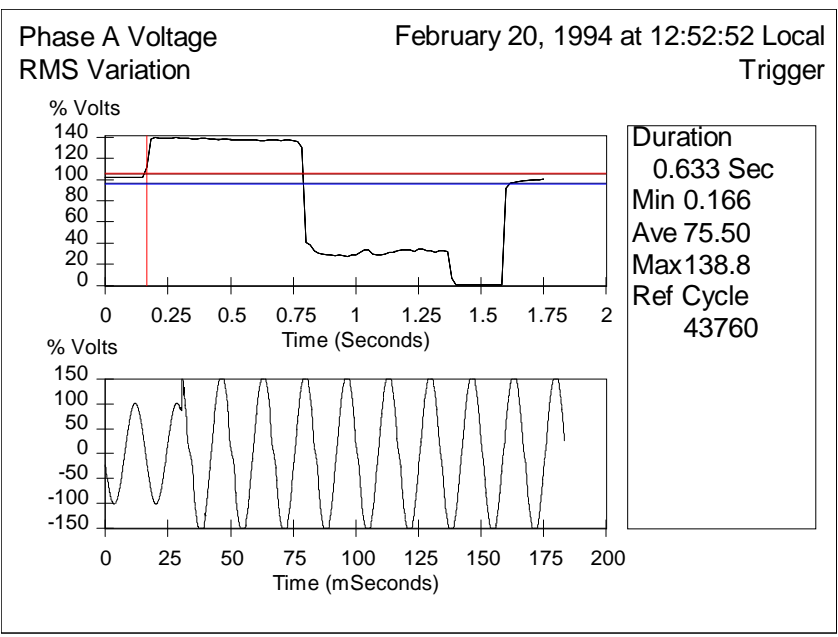

Fig.1. Non-rectangular rms variation.

Many different methods have been proposed for characterizing rms variation phase measurements. Most of these methods agree that the magnitude must be the maximum deviation magnitude of the measurement. The difficulty lies in assigning a duration associated with the magnitude. The method defined for calculating the indices presented in this paper is called the "Specified Voltage" method. This method designates the duration as the period of time that the rms voltage exceeds a specified threshold voltage level used to characterize the disturbance. Thus, a given non-rectangular event could be assigned different duration values depending on the specified voltage threshold being used for assessment. For example, the duration of the voltage reduction portion of Figure 1 would vary depending on the rms variation voltage level of interest. Figure 2 illustrates this concept for three voltage levels; $80 \%, 50 \%$, and $10 \% . \mathrm{T}_{80 \%}$ is the characterized duration of the event for 
an assessment of sags having magnitudes $\leq 80 \%$. Likewise, $\mathrm{T}_{50 \%}$ and $\mathrm{T}_{10 \%}$ are the durations associated with assessments of sags of the specified voltage levels. Notice that $\mathrm{T}_{80 \%}$ and $\mathrm{T}_{50 \%}$ are both $800 \mathrm{~ms}$ because both of the sag components of this non-rectangular event have magnitudes well below $50 \%$. $\mathrm{T}_{10 \%}$, however, comprises only the duration of the second component, 200 milliseconds.

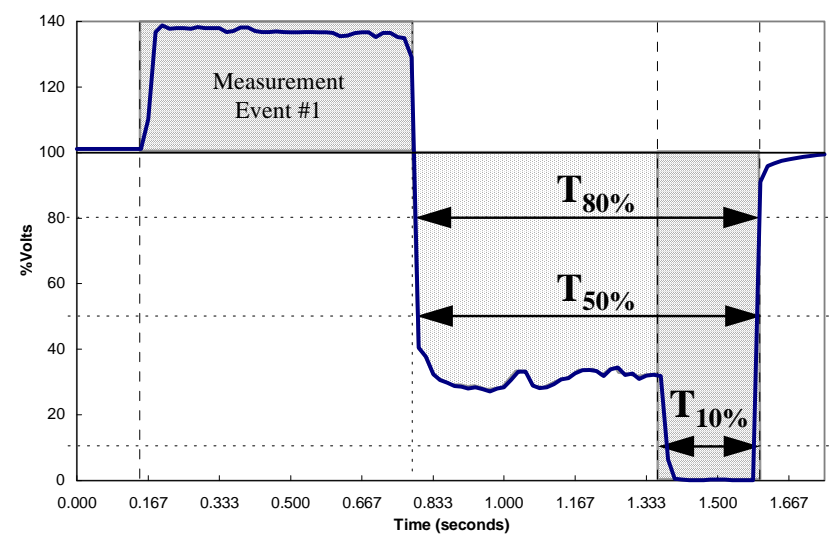

Fig.2. Illustration of "Specified Voltage" characterization of rms variation phase measurements.

Measurement Event Level. A power system occurrence such as a fault can affect one, two, or all three phases of the distribution system. Furthermore, the magnitude and duration of the resulting rms variation on each phase may differ substantially. When calculating rms variation indices, a determination must be made concerning how to report three-phase measurement events. Unfortunately, as with the characterization of non-rectangular components, characterization at the measurement event level has not been standardized and is not very well defined [4]. For an assessment of single-phase performance, each of the three phases are reported separately. Thus, for a three-phase fault, three different rms variations are included in the indices. The method of characterizing measurement events for the indices presented here is a three-phase method. A single set of characteristics are determined for all of the affected phases. For each rms variation measurement, the magnitude and duration are designated as the magnitude and duration of the phase with the greatest voltage deviation from nominal voltage.

Aggregate Event Level. An aggregate event is the collection of all measurements associated with a single power system occurrence into a single set of event characteristics. For example, a single distribution system fault might result in several measurements as the circuit protection system sequences through predefined operations. The aggregate event associated with this fault would summarize all of the associated measurements into a single set of characteristics (magnitude, duration, etc.). Many customer devices and processes trip or mis-operate on the initial rms variation associated with a fault. Once a device has shut down, the succeeding rms variations associated with the operation of protective devices have no adverse affect on customer process. Consequently, including multiple rms variations resulting from the same power system occurrence may distort the representation of the true state of the system service quality. Thus, aggregation provides a truer assessment of service quality with regard to many customers. The rms variation performance indices presented here are based on aggregate events.

Without the aid of an artificial intelligence algorithm, a good method of aggregating measurements is to use time: once the first measurement event has been identified, all measurements recorded by the instrument over the next defined duration are considered part of the same aggregate period. The data aggregation period chosen for the rms variation indices is one minute which correlates to the IEEE Std. 1159-1995 definition of the minimum length of a sustained interruption. Other aggregation periods could be used but the one-minute period is as appropriate as any other.

Using a one-minute aggregation period, the magnitude and duration of the aggregate event is designated to be the magnitude and duration of the measurement event most likely to result in customer equipment failure. The "worst" measurement event is assumed to be the event exhibiting the greatest voltage deviation.

\section{RMS VARIATION PERFORMANCE INDICES}

The developed rms variation indices are designed to aid in the assessment of service quality for a specified circuit area. The indices are defined such that they may be applied to systems of varying size. For example, the indices may be applied to measurements recorded across a utility's entire distribution system resulting in SAIFI-like system averages, or the indices may be applied to a smaller segment of the distribution system, such as a single feeder or a single customer point-of-common-coupling (PCC). As a result of this scalability of the indices, values can be calculated for various parts of the distribution system and compared to values calculated for the entire system.

There are many properties of rms variations which would be useful to quantify -- properties such as the frequency of occurrence, the duration of disturbances, the number of phases involved, etc. Consequently, many rms variation indices have been defined addressing these various issues. Only a subset of these indices are presented. The indices presented are those which are already being used by utilities such as United Illuminating to address service quality issues.

The susceptibility of various customer devices and processes to rms variations differs [5,6]. Many devices are susceptible to only the magnitude of the variation. Others are susceptible to the combination of magnitude and duration. Accordingly, the four indices presented assess rms variation magnitude and the combination of magnitude and duration. 
System Average RMS (Variation) Frequency Index ${ }_{\text {Voltage }}$ $\left(\boldsymbol{S A R F I}_{X}\right)$. SARFI $I_{x}$ represents the average number of specified rms variation measurement events that occurred over the assessment period per customer served, where the specified disturbances are those with a magnitude less than $x$ for sags or a magnitude greater than $x$ for swells.

$$
\operatorname{SARFI}_{x}=\frac{\sum N_{i}}{N_{T}}
$$

where

$x \equiv$ rms voltage threshold; possible values - 140, 120 , $110,90,80,70,50$, and 10

$N_{i} \equiv$ number of customers experiencing short-duration voltage deviations with magnitudes above $\mathrm{X} \%$ for $\mathrm{X}>100$ or below $\mathrm{X} \%$ for $\mathrm{X}<100$ due to measurement event $i$

$N_{T} \equiv$ number of customers served from the section of the system to be assessed

Notice that SARFI is defined with respect to the voltage threshold $x$. This allows for an assessment of rms variations of a specified voltage level. For example, if a utility has customers which are only susceptible to sags below $70 \%$ of nominal voltage, this disturbance group can be assessed using $S A R F I_{70}$. All of the rms variation indices are defined using this voltage threshold. Note also, that the 8 defined values for the index voltage threshold are not arbitrary values. They are chosen to coincide with the following: 140, 120, and 110: Overvoltage segments of new guidelines proposed for information technology equipment

90, 80, and 70: Undervoltage segments of new guidelines proposed for information technology equipment

50: Typical break point for assessing motor contactors

10: IEEE Std. 1159 definition of an interruption.

This group of indices is similar to the System Average Interruption Frequency Index (SAIFI) value that many utilities have calculated for years. $S A R F I_{X}$, however, assesses more than just interruptions. The frequency of occurrence of rms variations of varying magnitudes can be assessed using $S A R F I_{x}$. Note that $S A R F I_{x}$ is defined for shortduration variations as defined by IEEE 1159 and listed in Table 1. Other indices not discussed in this paper are defined for long-duration variations, undervoltages and overvoltages.

The following three indices are actually subsets of $S A R F I_{x}$. These indices assess variations of a specified magnitude and IEEE 1159 duration category.

System Instantaneous Average RMS (Variation) Frequency Index $_{\text {Voltage }}\left(\right.$ SIARFI $\left._{X}\right)$. SIARFI $I_{X}$ represents the average number of specified instantaneous rms variation measurement events that occurred over the assessment period per customer served. The specified disturbances are those with a magnitude less than $x$ for sags or a magnitude greater than $x$ for swells and a duration in the range of 0.5 30 cycles [1].

$$
\operatorname{SIARFI}_{X}=\frac{\sum N I_{i}}{N_{T}}
$$

where

$$
\begin{aligned}
& x \equiv \text { rms voltage threshold; possible values - } 140,120 \text {, } \\
& 110,90,80,70 \text {, and } 50 \\
& N I_{i} \equiv \text { number of customers experiencing instantaneous } \\
& \text { voltage deviations with magnitudes above } \mathrm{X} \% \text { for } \\
& \mathrm{X}>100 \text { or below } \mathrm{X} \% \text { for } \mathrm{X}<100 \text { due to } \\
& \text { measurement event } i
\end{aligned}
$$

Notice that $\operatorname{SIARFI}_{x}$ is not defined for a threshold value of $x=10$. This is because IEEE Std. 1159 does not define an instantaneous duration category for interruptions[1].

System Momentary Average RMS (Variation) Frequency Index $_{\text {Voltage }}\left(\operatorname{SMARFI}_{x}\right)$. In the same way that SIARFI $I_{x}$ is defined for instantaneous variations, $S M A R F I_{X}$ is defined for variations having durations in the range of 30 cycles to 3 seconds for sags and swells and in the range of 0.5 cycles to 3 seconds for interruptions [1].

$$
\operatorname{SMARFI}_{x}=\frac{\sum N M_{i}}{N_{T}}
$$

$x \equiv$ rms voltage threshold; possible values - 140,120 , 110, 90, 80, 70, 50, and 10

$N M_{i} \equiv$ number of customers experiencing momentary voltage deviations with magnitudes above $\mathrm{X} \%$ for $X>100$ or below $X \%$ for $X<100$ due to measurement event $i$

System Temporary Average RMS (Variation) Frequency Index $_{\text {Voltage }}\left(\boldsymbol{S T A R F I}_{x}\right)$. STARFI $I_{x}$ is defined for temporary variations which have durations in the range of $3-60$ seconds [1].

$$
\operatorname{STARFI}_{x}=\frac{\sum N T_{i}}{N_{T}}
$$

$x \equiv$ rms voltage threshold; possible values - 140, 120, $110,90,80,70,50$, and 10

$N T_{i} \equiv$ number of customers experiencing temporary voltage deviations with magnitudes above $\mathrm{X} \%$ for $\mathrm{X}>100$ or below $\mathrm{X} \%$ for $\mathrm{X}<100$ due to measurement event $i$

\section{EXAMPLE CALCULATED INDEX VALUES}

The definitions of the four service quality indices discussed may be difficult to assimilate without actual numbers being 
applied to the definitions. The following example demonstrates how the indices are calculated. The example is based on actual data recorded on one of the feeders monitored during a national power quality data collection effort [3].

Some assumptions are made in order to calculate the indices. By definition, in order to calculate the indices, one must know how many customers experience a voltage exceeding the index threshold for each rms variation that occurs. Obviously, every customer will not be individually monitored. Consequently, one must approximate the voltage experienced by each customer during a disturbance. This is accomplished by segmenting the circuit into areas across which the voltage is assumed to be uniform. Once the voltage is determined for a segment, it is assumed that all customers in the segment experience the same voltage. Obviously, the smaller the segments, the better the approximation.

One method of determining voltages for many circuit segments based on a limited number of monitoring points is distribution state estimation. State estimation provides pseudo-measurements for those segments not containing a measuring instrument. Disturbance state estimation requires a detailed circuit model and specified monitored data. State estimation tools are being developed which provide very reasonable results for individual circuit segments based on data from a limited number of actual monitoring points within the circuit.

Without the pseudo-measurements provided by state estimation, the assessed system must be segmented so that every point in the system is contained within a section monitored by an actual power quality measuring instrument. Thus, the number of monitoring locations within the assessed system becomes the number of constant voltage segments upon which the indices are calculated. Because this process of monitor-limited segmentation (MLS) results in only a few segments per circuit, the calculated index values are less accurate than those calculated using state estimation concepts. Nonetheless, MLS still yields indices which are informative. Figure 2 illustrates the MLS segments for the example calculation feeder. Note that there are three uniform voltage segments corresponding to the three power quality monitors, M1, M2, and M3. These MLS segments are the basis for the calculated values presented below. The number of customers served from each MLS segment was not available so arbitrary number of customer values of 500,100, and 400 were assumed for segments 1, 2, and 3, respectively. Based on the segmentation of Figure 3 and the assumed number of customers data, the rms variation indices were calculated based on one year of monitoring data. The results are summarized in Table 2.

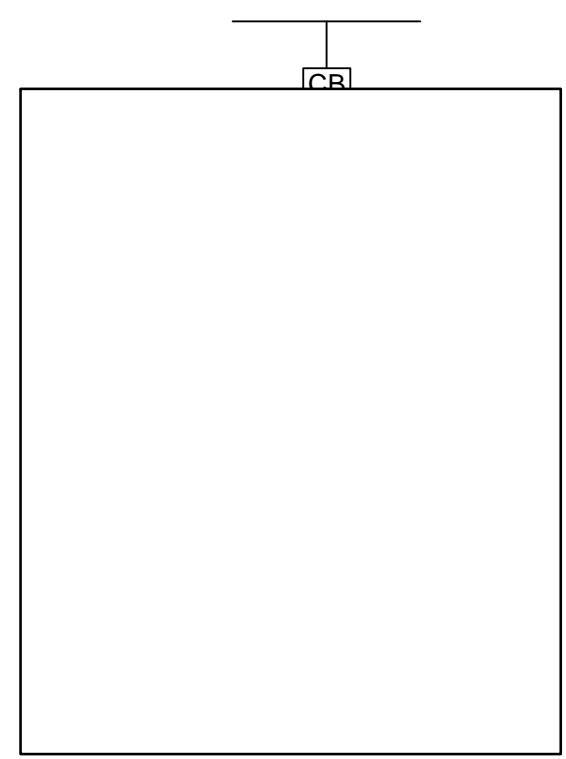

Fig.3. Circuit for which example rms variation indices are calculated.

\begin{tabular}{|c|c|c|c|c|}
\hline$x$ & $S A R F I_{x}$ & $S_{A A R F I}$ & $S$ & \\
\hline 140 & 0.0 & 0.0 & 0.0 & 0.0 \\
\hline 120 & 0.0 & 0.0 & 0.0 & 0.0 \\
\hline 110 & 0.5 & 0.5 & 0.0 & 0.0 \\
\hline 90 & 27.5 & 22.7 & 4.3 & 0.5 \\
\hline 80 & 13.6 & 8.8 & 4.3 & 0.5 \\
\hline 70 & 7.3 & 2.5 & 4.3 & 0.5 \\
\hline 50 & 4.8 & 0.5 & 3.8 & 0.5 \\
\hline 10 & 4.3 & Undefined & 3.8 & 0.5 \\
\hline
\end{tabular}

Table 2. Example rms variation index values calculated for circuit of Figure 1 based on 1 year of actual monitored data.

Although the indices of Table 2 were calculated using assumed number of customer data, the measurement data is real. Furthermore, as equations 1-4 indicate, the number of customers information is both the numerator and denominator of the indices. Consequently, the calculated values are logical. There are a very few swells. The $S A R F I_{110}$ value is 0.5 customer disturbances per customer served per year.

The sag indices are also typical of what would be expected. The number of customer disturbances decrease as the voltage threshold decreases. Of the 27.5 customer sags per customer served per year, only 7.3 are below $70 \%$ and only 4.8 are below $50 \%$. In correlation with a knowledge of the susceptibility of sensitive customers on this system, this information is useful in determining potential problems. Note that by using this monitored data, a $S A R F I_{10}$ value of 4.3 short-duration interruptions per customer served per year is calculated. Interruption indices such as SAIFI are often calculated using interruption device information and not monitored data. This value of 4.3 can not be compared to a SAIFI value, however, because SAIFI is based only on sustained interruptions. 
The duration based indices are also quite interesting. As expected, the majority of the disturbances are instantaneous as classified by IEEE 1159 . Only 4.8 of the 27.5 sag disturbances are either momentary or temporary. Notice that this is only true for the less severe magnitude sags (90\%, $80 \%$ and $70 \%$ ). The majority of the more severe sags (magnitude of $50 \%$ and less) are longer duration sags.

\section{PRESENT UTILITY APPLICATIONS}

Utilities are using the discussed rms variation indices to improve their systems. United Illuminating Co. (UI) computes the indices for individual substations. The indices are also calculated aggregately for several substations to create a system index. The individual substation values are then compared to the system value. Those substations that exhibit significantly poor performance as compared to the system performance are targeted for maintenance efforts. Based on the sensitivity and needs of the customers served from the targeted substations, the economic viability of potential mitigating actions are assessed. UI is also using the indices to relate system performance in a simplified manner to key industrial customers.

\section{CONCLUSIONS}

There is a recognized need among electricity distribution companies to assess the state of service being provided to customers on their systems. The discussed rms variation indices provide a means of quantifying such assessments and a common terminology for discussion. At least two US utilities are currently using the indices for various service quality assessments.

\section{REFERENCES}

[1] IEEE Std.1159-1995, Recommended Practice on Monitoring Electric Power Quality, Working Group on Monitoring Electrical Power Quality of SCC22 - Power Quality, Draft 6, November, 1994.

[2] IEEE P1366, Trial Use Guide for Electric Power Distribution Reliability Indices, Working Group on System Design, Draft 13, January, 1996.

[3] EPRI TR-106294-V2, An Assessment of Distribution System Power Quality; Volume 2: Statistical Summary Report, May 1996.

[4] ANSI/IEEE Std. 493-1980, IEEE Recommended Practice For Design of Reliable Industrial and Commercial Power Systems, 6 ${ }^{\text {th }}$ Printing, 1989.

[5] V. Wagner, A. Andreshak, and J. Staniak, "Power Quality and Factory Automation, " Proceedings of the IAS Annual Meeting, vol. 35, no. 6, pp. 13911396.

[6] M. F. McGranaghan, D. R. Mueller, M. J. Samotyj, "Voltage Sags in Industrial Systems," IEEE Transactions on Industry Applications, Vol 29, No. 2, March/April 1993.

\section{AUTHOR BIOGRAPHIES}

Daniel L. Brooks is a Power Systems Engineer with Electrotek Concepts in Knoxville, TN. He received his BS and MS degrees in Electrical Engineering from Mississippi State University in 1993 and 1994, respectively. He is presently serving as lead engineer for the EPRI Reliability Benchmarking Methodology Project. Prior to this project, he was actively involved in power quality data analysis for the EPRI Distribution Power Quality Project (DPQ).

Roger C. Dugan is Senior Consultant with Electrotek Concepts in Knoxville, TN. Prior to joining Electrotek in 1992, he worked 19 years for Cooper Power Systems. He received his BSEE from Ohio University in 1972 and his MSEE from Rensselaer Polytechnic Institute in 1973. His work has largely centered on computer simulations of distribution systems, particularly on power quality topics.

Marek Waclawiak is an electrical engineer with the United Illuminating Company in New Haven, CT. Marek received his MSEE from Politaechnic of Lodz in 1976. Marek manages UI's distribution power quality efforts including the power quality monitoring system. He is responsible for the collection, storage, and analysis of power quality data recorded at both primary distribution and secondary customer locations. Marek is a registered professional engineer in the state of Connecticut.

Ashok Sundaram is the Project Manager for Power Electronics in the Electric Power Research Institute's Distribution Power Delivery Group in Palo Alto, California. He directs $R \& D$ projects in the areas of power electronics and power quality. Ashok holds a BSEE from the University of Madras, India and a MSEE from Southern Illinois University. 